\title{
Short-term effects of ambient (outdoor) air pollution on cardiovascular death in Tehran, Iran - a time series study
}

\author{
Azizallah Dehghan ${ }^{\mathrm{a}}$, Narges Khanjani ${ }^{\mathrm{b}, \mathrm{c}}$, Abbas Bahrampour ${ }^{\mathrm{d}}$, Gholamreza Goudarzi ${ }^{\mathrm{e}, \mathrm{f}}$ and \\ Masoud Yunesian ${ }^{g, h}$
}

\begin{abstract}
${ }^{a}$ Neurology Research Center, Kerman University of Medical Sciences, Kerman, Iran; ${ }^{b}$ Environmental Health Engineering Research Center, Kerman University of Medical Sciences, Kerman, Iran; ${ }^{C}$ Monash Centre for Occupational \& Environmental Health, School of Public Health and Preventive Medicine, Monash University, Melbourne, Australia; ${ }^{d}$ Physiology Research Center \& Department of Biostatistics and Epidemiology, Faculty of Health, Kerman University of Medical Sciences Kerman, Iran; ${ }^{\text {e} A i r ~ P o l l u t i o n ~ a n d ~ R e s p i r a t o r y ~}$ Diseases Research Center, Ahvaz Jundishapur University of Medical Sciences, Ahvaz, Iran; ${ }^{f}$ Environmental Technologies Research Center (ETRC), Ahvaz Jundishapur University of Medical Sciences, Ahvaz, Iran; ${ }^{9}$ Department of Environmental Health Engineering, School of Public Health, Tehran University of Medical Sciences, Tehran, Iran; ${ }^{h}$ Center for Air Pollution Research \& Department of Research Methodology and Data Analysis, Institute for Environmental Research (IER), Tehran University of Medical Sciences,

Tehran, Iran
\end{abstract}

\section{ABSTRACT}

The aim of this study was to estimate the effect of ambient air pollutants on cardiovascular deaths in Tehran, Iran. In this time series study, air pollutant data were acquired from the Environmental Protection Agency. Meteorological data were acquired from the meteorological organization, and death data were acquired from the Tehran's cemetery registration. Generalized Additive Models (GAM) were used for estimating the Rate Ratio. $\mathrm{NO}_{2}, \mathrm{SO}_{2}$ and $\mathrm{PM}_{10}$ were associated with total cardiovascular deaths. $\mathrm{PM}_{10}$ and $\mathrm{NO}_{2}$ showed stronger relations with deaths in the elder age group. The result of this study showed that $\mathrm{NO}_{2}, \mathrm{SO}_{2}, \mathrm{PM}_{10}$ and $\mathrm{O}_{3}$ are probably responsible for part of the cardiovascular deaths that happen daily in Tehran.
ARTICLE HISTORY

Received 26 December 2017

Revised 10 June 2018

Accepted 10 June 2018

\section{KEYWORDS}

Air pollution; cardiovascular death; particulate matter; Tehran

\section{Introduction}

Air pollution is composed of a heterogeneous mixture of compounds including carbon monoxide (CO), ozone (O3), nitrogen oxides (NOx), sulfur dioxide (SO2), particulate matter (PM) and liquids (Sun et al. 2010).

Air pollution is a main environmental hazard and a threat to human health. The rising health consequences of air pollution have attracted the attention of researchers in the last decades. In 2014, 91\% of the global population lived in places where the levels of air pollutants failed to meet the World Health Organization's air quality standards. The World Health Organization (WHO) reported that in 2016 around 4.2 million people lost their lives because of inhaling outdoor polluted air. These facts confirm that air pollution is now the world's largest single environmental health risk (WHO, 2018).

Air pollution in developing countries is mainly related to their increased population, wrong ways of regulating vehicles, vast usage of fossil fuels, urban sprawl, immigration to big cities and inappropriate expansion of industries without appropriate site selection (Roushan et al. 2009, Rezaei et al. 2016).

Numerous epidemiological studies across the world, especially in developed countries have reported a relation between short-term changes in air pollution, and cardiovascular diseases and death especially in America, Europe and China (Wong et al. 2002, Stieb et al. 2003, Zhang et al. 2006, Lopez-Villarrubia et al. 2010, Sun et al. 2010, Yu et al. 2012, Yamamoto et al. 2014). A number of studies have also been conducted in Iran (Hosseinpoor et al. 2005, Shahi et al. 2014, Hashemi et al. 2014, Dadbakhsh et al. 2016, Hashemi and Khanjani 2016, Vahedian et al. 2017, Dastoorpoor et al. 2018, Dastoorpoor et al. 2019).

Although the Rate Ratio of mortality caused by air pollution is low, the proportion of deaths related to air pollution is high because of the high number of exposed and sensible populations (Qorbani and Yunesian 2010). Air pollution shows a nations environmental health and quality control problems. Although there are many studies done in the world about air

CONTACT Narges Khanjani $\_$n_khanjani@kmu.ac.ir $@$ Department of Epidemiology and Biostatistics, School of Public Health, Kerman University of Medical Sciences, Kerman, Iran

(C) 2019 Informa UK Limited, trading as Taylor \& Francis Group 
pollution and its health effects, but there have been fewer studies from developing countries; and, therefore, studying the various aspects of air pollution in developing countries is essential (Santus et al. 2012).

Tehran is the capital and the biggest city of Iran, with a population of about 8.5 million people, from the 80 million people who live in Iran. The area of Tehran is $1500 \mathrm{~km}^{2}$. This city is very densely populated and suffers from air pollution due to its specific geographical conditions (topography and meteorology), social and cultural problems (population distribution, traffic), urban development and abundant consumption of energy in transportation and industry. Controlling air pollution has been complicated in Tehran and investigation about the different aspects of air pollution and its health effects is still necessary (Hosseinpoor et al. 2005, Khalilzadeh et al. 2009).

Given the importance of air pollution in Tehran, this time series study was carried out to find about the short-term effects of air pollution on deaths due to cardiovascular disease in this city.

\section{Methods}

This was a time series and population-based study conducted in Tehran, Iran. Tehran is geographically located in a valley and surrounded by medium to high mountains on its north, northwest, east, and southeast.

\section{Air pollution, meteorological and death data}

Data on ambient air pollutants $\left(\mathrm{CO}, \mathrm{O}_{3}, \mathrm{NO}_{2}, \mathrm{SO}_{2}\right.$, and $\mathrm{PM}_{10}$ ) from 2005 to 2014 were collected from the Tehran Department of Environment and Tehran Air Quality Control Company. Tehran has 22 municipality districts and there are one or more air pollution monitoring stations in each district (36 in total). In all monitoring stations, the concentrations of air pollutants are registered hourly. In this study, the outlier observations were identified with spatio-temporal screening tools. In this method the mean and standard deviation of each observation in a specific hour, and 1 and $2 \mathrm{~h}$ before and after it, in each station and the neighboring stations were computed. Then observation which were more than \pm 3 SD away from the mean were recognized as outliers (Shamsipour et al. 2014, Dehghan et al. 2018).

Missing air pollution data was estimated using the Expectation-Maximization (EM) algorithm in SPSS20 software. This approach was proposed by Dempster et al. (1977). This method is an algorithm for nonlinear optimization and is appropriate for time series applications involving unknown components (Anava et al. 2015). EM is a repetitive and effective process that uses maximum likelihood estimation in estimating missing data. Each repetition of the algorithm consists of two steps: the mathematical expectation stage (EStep) and the maximization step (M-Step). In the mathematical expectation step, missing data is estimated based on observed data and the current estimation of the model parameters. In the maximization step, the likelihood function is maximized with the assumption that the missing data is known. Here, the estimates of missing data from the E-step are placed instead of missing values. By repeating the algorithm, the missing value is corrected at each step, and convergence can be assured (Afshari Safavi et al. 2015).

After missing imputation was done, the daily averages of pollutants in selected stations were computed. Then the average of included stations was computed and one value was generated for the whole city in all days. The included stations were the Aghdasiyeh, Azadi, Poonak, Pardisan, Razi, Park-e-roz and Shahr-erey stations.

The data of cardiovascular deaths were collected from the Tehran's Cemetery (Behesht-e Zahra) Organization. Cardiovascular deaths included deaths from heart attacks, strokes, heart diseases related to blood pressure, kidney diseases related to blood pressure, pulmonary embolism, embolism and arterial thrombosis, aortic aneurysm, other vascular diseases, other heart diseases, other cardiovascular diseases, non-rheumatic disorders in the mitral and aortic valves, acute and sub-acute endocarditis, acute pericarditis, acute myocarditis, cardiomyopathy, cardiac failure, and congenital abnormality of the cardiovascular system. The daily count of cardiovascular deaths was entered in the GAM model as the outcome variable.

The daily average of meteorological data (temperature and relative humidity), were inquired from the Tehran Meteorological Organization as potential confounder variables; because some studies have reported that cardiovascular disease mortality may change with fluctuations in temperature (Khanjani and Bahrampour 2013), although other studies have denied a relation (Dadbakhsh et al. 2018).

\section{Data analysis}

The mean, standard deviation, median, $25^{\text {th }}$ and $75^{\text {th }}$ percentiles of air pollutants, meteorological variables 
and the frequency of cardiovascular deaths were computed.

A time-series regression analysis was used to assess the short-term association between air pollutant exposures and count of cardiovascular deaths. Generalized Additive Models (GAM) were used to estimate Rate Ratio (RR). GAM models have been widely used in studies about air pollution and health outcomes; because they are able to adjust for the effect of nonlinear confounding variables such as seasonal changes, trends and meteorological variables (Dominici et al. 2002, Guisan et al. 2002, Dehghan et al. 2018).

The degree of freedom for the smoothers was determined by Generalized Cross Validation (GCV) using the "mgcv" package in R i386 3.2.2 software.

Multivariate GAM models were also run for the same outcome and in subgroups. Relative humidity, temperature, season and weekdays were entered into the model as potential confounding variables. Then the strongest lag for pollutants was reported.

The formula of the GAM model is as follows (Wang and Pham 2011).

$$
\begin{aligned}
& Y_{t} \sim \text { Poisson }\left(\mu_{t}\right) \\
\log \mu_{t}= & \alpha+\beta_{i}\left(X_{i}\right) \\
& +\sum S_{j}\left(X_{j}\right)+\mathrm{y} S(\text { season })+\eta D(\text { dow })
\end{aligned}
$$

In this formula $Y_{t}$ is the frequency of the incidence of cardiovascular deaths. $\beta_{i}$ is the coefficient for air pollutants $\left(X_{i}\right)$ and indicates the logarithm of Risk Ratio (RR) for 10 unit increase in all pollutants $(10 \mu \mathrm{g} /$ $\mathrm{m}^{3}$ for $\mathrm{PM}_{10}$ and $10 \mathrm{ppb}$ for $\mathrm{O}_{3}, \mathrm{SO}_{2}$ and $\mathrm{NO}_{2}$ ), except $\mathrm{CO}$ which it is for $1 \mathrm{ppm}$ increase. Furthermore, $\mathrm{S}_{j}\left(\mathrm{X}_{j}\right)$ is a smoothing function for meteorological variables (relative humidity and temperature) and trend. $S$ (season) are indicator variables for Spring, Summer, Autumn and Winter. D(dow) are indicator variables for weekdays. Season and weekdays were added to the model as categorical variables.

In this study, due to the correlation between pollutants, one pollutant models were performed. But, we also used two pollutant models to assess the stability of the results from the one pollutant models.

\section{Result}

During the 10-year study period, 215373 cardiovascular deaths occurred in Tehran which included 122911 (57.07\%) male and 92462 (42.93\%) female deaths. The frequencies of cardiovascular deaths are shown in Table 1 by year and gender. Figure 1 shows the trend of cardiovascular deaths.
Table 1. Count of deaths due to cardiovascular disease in Tehran during 2005-2014 by sex and year.

\begin{tabular}{lrrcrc}
\hline Year & Male & Female & Male/Female & Total & ASR $^{*}$ \\
\hline 2005 & 12,724 & 9449 & 1.34 & 22,173 & 277.99 \\
2006 & 11,757 & 8767 & 1.34 & 20,524 & 258.96 \\
2007 & 11,975 & 8894 & 1.34 & 20,869 & 259.03 \\
2008 & 12,916 & 9567 & 1.35 & 22,483 & 269.63 \\
2009 & 12,190 & 9100 & 1.34 & 21,290 & 256.58 \\
2010 & 12,393 & 9332 & 1.32 & 21,725 & 257.31 \\
2011 & 12,281 & 9279 & 1.32 & 21,560 & 253.72 \\
2012 & 11,358 & 8629 & 1.31 & 19,987 & 238.01 \\
2013 & 12,426 & 9526 & 1.30 & 21,952 & 252.89 \\
2014 & 12,891 & 9919 & 1.29 & 22,810 & 258.1 \\
Total & 122,911 & 92,462 & 1.32 & 215,373 & - \\
\hline
\end{tabular}

*Age- standardized cardiovascular rate per 100,000 population.

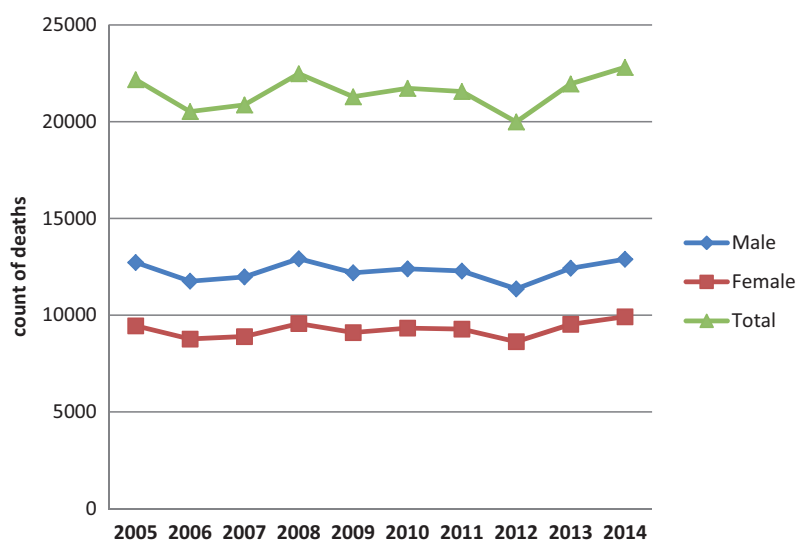

Figure 1. The frequency of cardiovascular deaths between 2005 and 2014.

Figure 2 shows the scatter plot of air pollutants and death during 2005 to 2014 in Tehran.

The descriptive statistics of air pollutants, humidity, temperature and death during 2005-2014 in Tehran are showed in Table 2 . These results show the annual average of $\mathrm{PM}_{10}$ was over the WHO 2014 annual thresholds guidelines $\left(20 \mu \mathrm{g} / \mathrm{m}^{3}\right)$. The average of $\mathrm{SO}_{2}$ concentrations was higher than the standard values as well.

Table 3 shows the Mean \pm SD of pollutants and number of cardiovascular deaths by season in Tehran, during 2005-2014. The highest concentration of $\mathrm{O}_{3}$ was in summer, $\mathrm{CO}$ and $\mathrm{PM}_{10}$ was in fall, and $\mathrm{NO}_{2}$ and $\mathrm{SO}_{2}$ was in winter and spring respectively. Also most cardiovascular deaths occurred in winter $(29.03 \%)$ and the lowest number of deaths was in summer (21.34\%).

Table 4 shows the correlation between different pollutants. All pollutants had a significant correlation with each other; except $\mathrm{O}_{3}$ that showed a direct correlation only with $\mathrm{PM}_{10}$, and an inverse correlation with $\mathrm{CO}$. The highest correlation was found between nitrogen dioxide and sulfur dioxide $(r=0.696$, $p=<.001)$. 


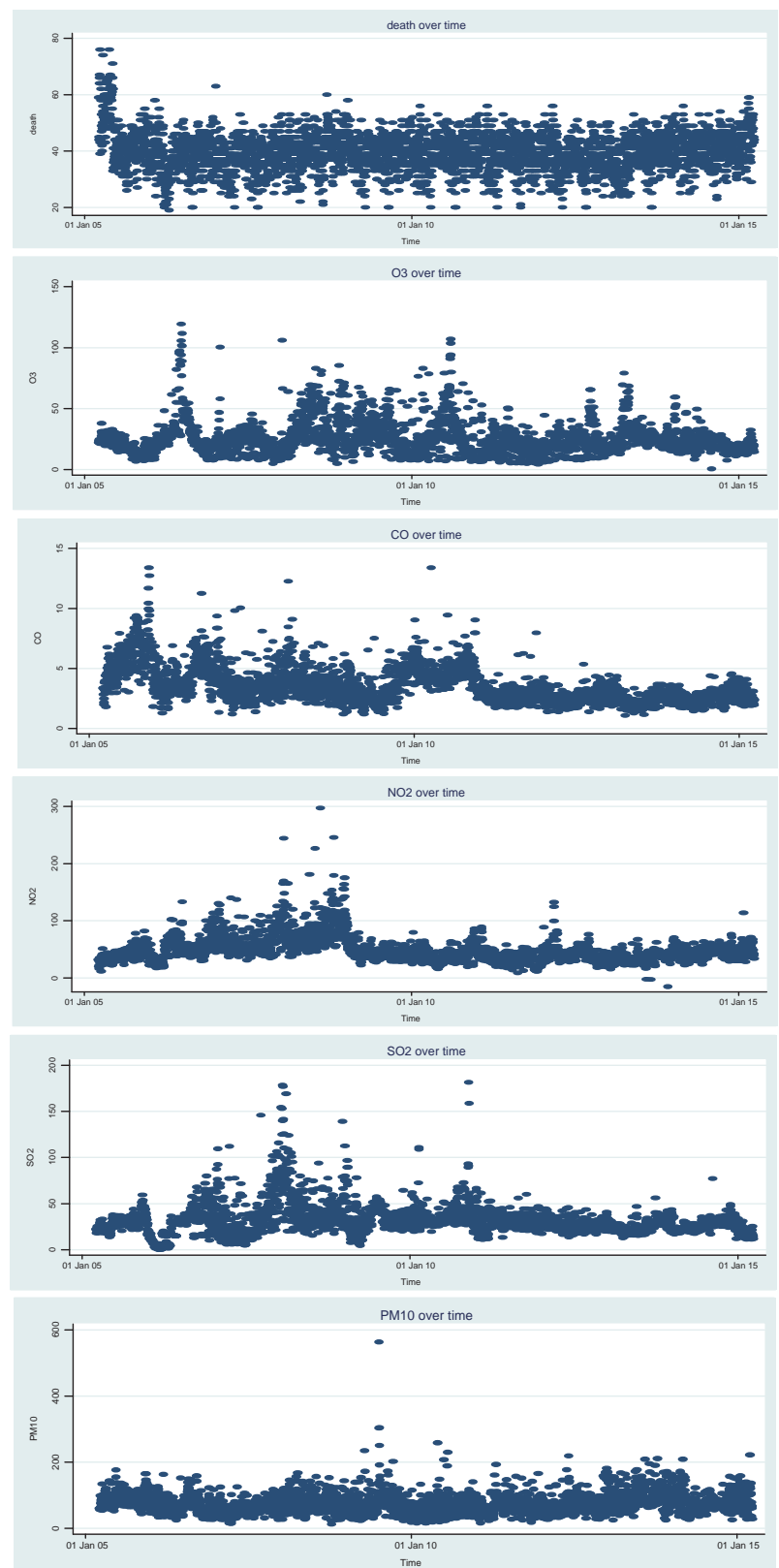

Figure 2. Scatter plot of total cardiovascular deaths and air pollutants during the study period (2005-2014).

Table 2. Mean, median, SD, min and max of daily air pollutants, meteorological and death variables in Tehran, 2005-2014.

\begin{tabular}{lrrrcr}
\hline & Mean & \multicolumn{1}{c}{ SD } & Median & Minimum & Maximum \\
\hline CO $(\mathrm{ppm})$ & 3.30 & 0.97 & 3.14 & 1 & 11 \\
O3 $(\mathrm{ppb})$ & 24.66 & 9.12 & 23.30 & 6.88 & 77 \\
SO2 $(\mathrm{ppb})$ & 33.46 & 10.47 & 32.05 & 9 & 119 \\
NO2 $(\mathrm{ppb})$ & 53.48 & 16.52 & 49.84 & 12 & 154 \\
PM10 $\left(\mu \mathrm{g} / \mathrm{m}^{3}\right)$ & 69.75 & 20.61 & 68.04 & 12 & 331 \\
Relative humidity $(\%)$ & 43.73 & 18.37 & 39.50 & 10 & 89 \\
Temperature $\left({ }^{\circ} \mathrm{C}\right)$ & 16.49 & 9.52 & 16.80 & -9 & 34 \\
Male deaths $(\mathrm{N})$ & 33.66 & 7.09 & 34.00 & 12 & 56 \\
Female deaths $(\mathrm{N})$ & 25.32 & 5.54 & 26.00 & 10 & 41 \\
Overal deaths $(\mathrm{N})$ & 58.97 & 9.36 & 59.00 & 30 & 94 \\
Under 18 years $(\mathrm{N})$ & 0.93 & 1.12 & 1 & 0 & 6 \\
18 to 60 years $(\mathrm{N})$ & 18.89 & 3.94 & 19 & 9 & 30 \\
Over 60 years $(\mathrm{N})$ & 39.16 & 6.25 & 38 & 19 & 63 \\
\hline
\end{tabular}

Table 5 shows the GAM results of air pollutants and total cardiovascular death. After adjusting for confounders including temperature, relative humidity, trend, season and DOW; total deaths were significantly associated with $\mathrm{NO}_{2}$ in lag 0 to lag 4 in the one pollutant model and lag 0 to 3 in the two pollutant model. But the strongest relation was seen in lag 3 in the one pollutant and lag 1 in the two pollutant model. $\mathrm{SO}_{2}$ in the one pollutant model showed significant associations with total death in lag 0 and 1 . But in the two pollutant model no significant relation was seen. $\mathrm{PM}_{10}$ showed a significant association with total death in lag 0 to 3 in both models (one and two pollutant). But the strongest relation was seen in lag 0 .

Table 6 shows the RR of air pollutants and cardiovascular deaths in men. In men, $\mathrm{NO}_{2}, \mathrm{SO}_{2}$ and $\mathrm{PM}_{10}$ had the strongest direct relation with cardiovascular death in lag 3, 0 and 0 respectively, in the one pollutant model. But in the two pollutant model, the strongest relation for $\mathrm{NO}_{2}$ and $\mathrm{PM}_{10}$ was seen in lag 0 ; and $\mathrm{SO}_{2}$ did not show a significant relation with death.

Table 7 shows the RR of air pollutants and cardiovascular deaths in women. In women, $\mathrm{O}_{3}$ showed a significant direct relation with deaths, in lag $0-3$ in the one pollutant and lag $0-4$ in the two pollutant models. $\mathrm{NO}_{2}$ in lag 6 (one pollutant model) and lag 5 (two pollutant model) had a significant relation with death. $\mathrm{PM}_{10}$ had a relation with cardiovascular deaths in lag $0-2$ in the one and two pollutant models.

No pollutant was related to cardiovascular deaths in people under 18 years old (Table 8 ). In people 18-60 years, $\mathrm{NO}_{2}, \mathrm{SO}_{2}$ and $\mathrm{PM}_{10}$ showed a strong relation with death in lag 1, 0 and 0 (day) respectively, in the one pollutant model. But in the two pollutant model, $\mathrm{NO}_{2}$ and $\mathrm{PM}_{10}$ showed the strongest relation in lag 3 (Table 9).

$\mathrm{NO}_{2}$ and $\mathrm{PM}_{10}$ in people over 60 years had the strongest relation with cardiovascular deaths in 1 , and 0 day lags respectively in the one and two pollutant models. $\mathrm{SO}_{2}$ showed a direct relation only in the one pollutant model (Table 10).

\section{Discussion}

This study showed a probable association between high levels of $\mathrm{NO}_{2}, \mathrm{SO}_{2}$, and $\mathrm{PM}_{10}$ with cardiovascular mortality.

In this study, with a $10 \mathrm{mg} / \mathrm{m}^{3}$ increase in $\mathrm{PM}_{10}$ on the same day, total cardiovascular deaths increased $0.57 \%$ (95\% Cl: $0.34-0.79 \%)$ in the one pollutant model and $0.59 \%(95 \% \mathrm{Cl}: 0.28-0.84 \%)$ in the two pollutant model. $\mathrm{PM}_{10}$ was also related with cardiovascular death 
Table 3. Mean \pm SD of pollutant and number of cardiovascular death by season in Tehran, 2005-2014.

\begin{tabular}{lcccccc}
\hline & $\mathrm{CO}$ & $\mathrm{O}_{3}$ & $\mathrm{NO}_{2}$ & $\mathrm{SO}_{2}$ & $\mathrm{PM}_{10}$ & Cont(\%) of death \\
\hline Spring & $2.95 \pm 0.80$ & $28.30 \pm 7.34$ & $48.21 \pm 14.16$ & $36.58 \pm 14.04$ & $66.40 \pm 21.62$ & $52056(24.17)$ \\
Summer & $3.28 \pm 0.89$ & $29.03 \pm 9.58$ & $51.22 \pm 12.74$ & $36.39 \pm 9.05$ & $68.74 \pm 18.12$ & $45961(21.34)$ \\
Fall & $3.59 \pm 1.13$ & $19.88 \pm 6.46$ & $56.99 \pm 16.60$ & $32.57 \pm 7.74$ & $76.88 \pm 20.42$ & $54834(25.46)$ \\
winter & $3.40 \pm 0.94$ & $21.13 \pm 8.81$ & $57.78 \pm 19.94$ & $28.51 \pm 7.75$ & $66.82 \pm 20.39$ & $62522(29.03)$ \\
\hline
\end{tabular}

Table 4. Pearson correlation coefficients between air pollutants.

\begin{tabular}{|c|c|c|c|c|c|}
\hline & 03 & $\mathrm{CO}$ & $\mathrm{NO}_{2}$ & $\mathrm{SO}_{2}$ & $\mathrm{PM}_{10}$ \\
\hline $\mathrm{O}_{3}$ & 1 & & & & \\
\hline $\mathrm{CO}$ & $-0.162^{* *}$ & 1 & & & \\
\hline $\mathrm{NO}_{2}$ & 0.019 & $0.414^{* *}$ & 1 & & \\
\hline $\mathrm{SO}_{2}$ & 0.067 & $0.473^{* *}$ & $0.696^{* *}$ & 1 & \\
\hline $\mathrm{PM}_{10}$ & $0.249 * *$ & $0.205^{* *}$ & $0.056^{*}$ & $0.263^{* *}$ & 1 \\
\hline
\end{tabular}

Table 5. Results of Adjusted Generalized Additive Model, about the effect of air pollutants on total cardiovascular death, for 1 unit increase in $\mathrm{CO}$ and 10 units increase in all other pollutants (adjusted for relative humidity, temperature, trend, season and day of week).

\begin{tabular}{|c|c|c|c|c|c|c|c|}
\hline & \multirow[b]{2}{*}{ lag } & \multicolumn{3}{|c|}{ One pollutant } & \multicolumn{3}{|c|}{ Two pollutant } \\
\hline & & $\mathrm{df}$ & RR $(95 \% \mathrm{Cl})$ & $p$ & $\mathrm{df}$ & RR $(95 \% \mathrm{Cl})$ & $p$ \\
\hline \multirow{8}{*}{$\mathrm{CO}(\mathrm{ppm})$} & 0 & 2.57 & $0.998(0.991-1.005)$ & .577 & 2.48 & $0.996(0.989-1.003)$ & .289 \\
\hline & 1 & 1.88 & $1.002(0.999-1.009)$ & .564 & 2.21 & $1.002(0.994-1.009)$ & .679 \\
\hline & 2 & 2.60 & $0.998(0.992-1.005)$ & .658 & 4.82 & $0.998(0.991-1.005)$ & .582 \\
\hline & 3 & 5.64 & $0.995(0.988-1.002)$ & .191 & 6 & $0.994(0.987-1.001)$ & .112 \\
\hline & 4 & 6.4 & $0.996(0.989-1.003)$ & .174 & 7.02 & $0.992(0.984-0.998)$ & .02 \\
\hline & 5 & 6.67 & $0.994(0.987-1.001)$ & .121 & 7.27 & $0.991(0.984-0.998)$ & .015 \\
\hline & 6 & 1 & $0.992(0.985-0.999)$ & .040 & 2.84 & $0.994(0.987-1.002)$ & .134 \\
\hline & 7 & 2.53 & $0.995(0.988-1.002)$ & .206 & 1 & $0.993(0.986-1.001)$ & .094 \\
\hline \multirow[t]{8}{*}{$\mathrm{O}_{3}(\mathrm{ppb})$} & 0 & 7.99 & $0.996(0.990-1.002)$ & .212 & 8.06 & $1.004(0.992-0.998)$ & .591 \\
\hline & 1 & 3.39 & $0.999(0.993-1.006)$ & .965 & 3.18 & $1.008(0.996-1.002)$ & .450 \\
\hline & 2 & 3.47 & $0.999(0.993-1.005)$ & .838 & 2.93 & $1.007(0.995-1.002)$ & .629 \\
\hline & 3 & 3.74 & $0.998(0.992-1.004)$ & .549 & 3.15 & $1.006(0.994-1.000)$ & .892 \\
\hline & 4 & 4.05 & $0.998(0.992-1.005)$ & .326 & 3.69 & $0.994(0.988-1.001)$ & .101 \\
\hline & 5 & 4.7 & $0.991(0.985-0.997)$ & .003 & 4.44 & $0.995(0.989-1.002)$ & .199 \\
\hline & 6 & 5.74 & $0.992(0.985-0.999)$ & .008 & 4.8 & $0.996(0.989-1.002)$ & .218 \\
\hline & 7 & 4.85 & $0.993(0.986-0.998)$ & .021 & 5.7 & $0.994(0.988-1.001)$ & .091 \\
\hline \multirow{8}{*}{$\mathrm{NO}_{2}(\mathrm{ppb})$} & 0 & 2.83 & $1.007(1.003-1.010)$ & $<.001$ & 2.37 & $1.005(1.002-1.008)$ & .003 \\
\hline & 1 & 1.59 & $1.007(1.004-1.011)$ & $<.001$ & 1 & $1.005(1.002-1.009)$ & .001 \\
\hline & 2 & 1 & $1.006(1.003-1.009)$ & $<.001$ & 1 & $1.005(1.001-1.008)$ & .004 \\
\hline & 3 & 1.28 & $1.008(1.003-1.009)$ & $<.001$ & 1.59 & $1.004(1.001-1.008)$ & .006 \\
\hline & 4 & 4.29 & $1.007(1.004-1.011)$ & $<.001$ & 4.66 & $1.009(0.998-1.013)$ & .165 \\
\hline & 5 & 2.32 & $1.008(0.999-1.018)$ & .128 & 3.8 & $1.003(0.999-1.006)$ & .286 \\
\hline & 6 & 1.76 & $1.004(0.995-1.013)$ & .255 & 1 & $1.002(0.998-1.005)$ & .275 \\
\hline & 7 & 1.33 & $1.007(1.003-1.010)$ & .306 & 1.02 & $1.001(0.998-1.005)$ & .246 \\
\hline \multirow[t]{8}{*}{$\mathrm{SO}_{2}(\mathrm{ppb})$} & 0 & 8.50 & $1.009(1.004-1.014)$ & $<.001$ & 8.18 & $1.001(0.995-1.007)$ & .681 \\
\hline & 1 & 8.41 & $1.007(1.002-1.012)$ & .009 & 1 & $0.997(0.992-1.004)$ & .483 \\
\hline & 2 & 8.32 & $1.005(0.999-1.010)$ & .069 & 1 & $0.998(0.992-1.004)$ & .596 \\
\hline & 3 & 1 & $1.004(0.999-1.009)$ & .095 & 1 & $1.001(0.994-1.006)$ & .818 \\
\hline & 4 & 4.05 & $1.002(0.997-1.007)$ & .283 & 1 & $1.003(0.997-1.009)$ & .253 \\
\hline & 5 & 1 & $1.005(0.999-1.010)$ & .059 & 1 & $1.002(0.995-1.007)$ & .678 \\
\hline & 6 & 1 & $1.004(0.998-1.009)$ & .153 & 1 & $0.999(0.993-1.005)$ & .913 \\
\hline & 7 & 1 & $1.004(0.999-1.009)$ & .164 & 1 & $1.002(0.996-1.008)$ & .356 \\
\hline \multirow[t]{8}{*}{$\mathrm{PM}_{10}\left(\mu \mathrm{g} / \mathrm{m}^{3}\right)$} & 0 & 1.88 & $1.005(1.003-1.008)$ & $<.001$ & 2.53 & $1.006(1.002-1.008)$ & $<.001$ \\
\hline & 1 & 1.69 & $1.006(1.003-1.008)$ & $<.001$ & 2.54 & $1.005(1.003-1.007)$ & $<.001$ \\
\hline & 2 & 7.44 & $1.004(1.001-1.006)$ & .001 & 7.38 & $1.003(1.001-1.005)$ & .002 \\
\hline & 3 & 6.83 & $1.002(1.000-1.005)$ & .043 & 6.39 & $1.003(0.999-1.005)$ & .043 \\
\hline & 4 & 6.20 & $1.002(0.997-1.006)$ & .389 & 6.38 & $1.000(0.997-1.003)$ & .838 \\
\hline & 5 & 6.95 & $1.001(0.998-1.003)$ & .467 & 2.27 & $1.001(0.999-1.004)$ & .25 \\
\hline & 6 & 6.83 & $1.002(0.999-1.004)$ & .078 & 6.66 & $1.002(0.999-1.004)$ & .091 \\
\hline & 7 & 6.84 & $1.002(0.999-1.004)$ & .051 & 6.44 & $1.017(0.994-1.041)$ & .134 \\
\hline
\end{tabular}

in men, women, and in the 18-60 and over 60 years old age groups. Similar to these results, in Wuhan, China, the maximum effect of $\mathrm{PM}_{10}$ on cardiovascular deaths occurred on the same day, and was a $0.51 \%$ increase for every $10 \mu \mathrm{g} / \mathrm{m}^{3}$ increase in $\mathrm{PM}_{10}(95 \% \mathrm{Cl}$ 0.28-0.75\%) (Qian et al. 2007). In a meta-analysis, the 
Table 6. Results of Adjusted Generalized Additive Model, about the effect of air pollutants on male cardiovascular death, for 1 unit increase in $\mathrm{CO}$ and 10 units increase in all other pollutants (adjusted for relative humidity, temperature, trend, season and day of week).

\begin{tabular}{|c|c|c|c|c|c|c|c|}
\hline & \multirow[b]{2}{*}{ lag } & \multicolumn{3}{|c|}{ One pollutant } & \multicolumn{3}{|c|}{ Two pollutant } \\
\hline & & df & $\mathrm{RR}(95 \% \mathrm{Cl})$ & $p$ & $d f$ & $\mathrm{RR}(95 \% \mathrm{Cl})$ & $p$ \\
\hline \multirow[t]{8}{*}{ CO (ppm) } & 0 & 2.34 & $1.005(0.995-1.014)$ & .319 & 2.56 & $0.999(0.989-1.008)$ & .846 \\
\hline & 1 & 2.30 & 1.009 (0.999-1.018) & .06 & 2.64 & $1.002(0.993-1.012)$ & .628 \\
\hline & 2 & 3.06 & $1.006(0.996-1.015)$ & .239 & 3.8 & $0.996(0.986-1.005)$ & .413 \\
\hline & 3 & 2.69 & $0.999(0.990-1.009)$ & .948 & 4.43 & $0.991(0.981-1.000)$ & .059 \\
\hline & 4 & 4.99 & $0.999(0.992-1.007)$ & .473 & 5.64 & $0.984(0.975-0.993)$ & .001 \\
\hline & 5 & 3.65 & $0.996(0.987-1.005)$ & .433 & 5.52 & $0.988(0.979-0.997)$ & .014 \\
\hline & 6 & 2.92 & $0.989(0.979-0.998)$ & .023 & 2.96 & $0.986(0.977-0.995)$ & .004 \\
\hline & 7 & 2.44 & $0.992(0.983-1.002)$ & .113 & 4.09 & $1.005(0.995-1.014)$ & .294 \\
\hline \multirow[t]{8}{*}{$\mathrm{O}_{3}(\mathrm{ppb})$} & 0 & 7.81 & $0.982(0.974-0.991)$ & $<.001$ & 7.88 & $0.986(0.978-0.994)$ & .001 \\
\hline & 1 & 6.80 & $0.987(0.979-0.996)$ & .002 & 2.46 & $0.991(0.983-1.000)$ & .051 \\
\hline & 2 & 2.95 & $0.988(0.980-0.996)$ & .003 & 2.90 & 0.991 (0.983-0.999) & .049 \\
\hline & 3 & 3.35 & $0.991(0.981-1.000)$ & .36 & 3.17 & $0.991(0.983-0.999)$ & .031 \\
\hline & 4 & 4.32 & $0.991(0.981-1.000)$ & 619 & 4.09 & $0.984(0.976-0.992)$ & .001 \\
\hline & 5 & 5.06 & $0.991(0.981-1.001)$ & .226 & 4.72 & $0.986(0.978-0.994)$ & .001 \\
\hline & 6 & 5.84 & $0.954(0.944-0.963)$ & $<.001$ & 6.15 & $0.987(0.979-0.996)$ & .003 \\
\hline & 7 & 6.25 & $0.983(0.975-0.991)$ & $<.001$ & 5.73 & $1.002(0.996-1.007)$ & .526 \\
\hline \multirow[t]{8}{*}{$\mathrm{NO}_{2}(\mathrm{ppb})$} & 0 & 2.85 & 1.009 (1.005-1.014) & $<.001$ & 2.64 & $1.010(1.005-1.015)$ & $<.001$ \\
\hline & 1 & 2.56 & $1.011(1.006-1.015)$ & $<.001$ & 2.97 & 1.009 (1.005-1.014) & $<.001$ \\
\hline & 2 & 2.57 & $1.010(1.005-1.014)$ & $<.001$ & 2.8 & $1.011(1.006-1.016)$ & $<.001$ \\
\hline & 3 & 2.41 & $1.011(1.006-1.015)$ & $<.001$ & 7.43 & 1.012 (1.007-1.017) & $<.001$ \\
\hline & 4 & 2.25 & $1.008(1.003-1.014)$ & $<.001$ & 7.41 & $1.004(0.999-1.006)$ & .126 \\
\hline & 5 & 1.81 & $1.010(1.005-1.015)$ & $<.001$ & 1.76 & $1.003(0.998-1.008)$ & .276 \\
\hline & 6 & 1.35 & $1.001(0.996-1.005)$ & .542 & 1 & 1.002 (0.999-1.005) & .143 \\
\hline & 7 & 1.05 & $1.004(0.995-1.012)$ & .289 & 1 & $1.003(0.998-1.007)$ & .052 \\
\hline \multirow[t]{8}{*}{$\mathrm{SO}_{2}(\mathrm{ppb})$} & 0 & 8.22 & $1.014(1.007-1.021)$ & $<.001$ & 7.71 & $0.998(0.990-1.006)$ & .640 \\
\hline & 1 & 8.17 & 1.009 (1.003-1.016) & .006 & 7.81 & $0.993(0.986-1.002)$ & .119 \\
\hline & 2 & 8.06 & $1.008(1.001-1.015)$ & .018 & 1.91 & $0.994(0.986-1.002)$ & .144 \\
\hline & 3 & 8.29 & 1.007 (1.001-1.014) & .032 & 1.79 & $0.995(0.988-1.004)$ & .305 \\
\hline & 4 & 1 & $1.004(0.995-1.013)$ & .114 & 1 & $0.995(0.988-1.003)$ & .285 \\
\hline & 5 & 1 & $1.006(0.999-1.013)$ & .09 & 1 & $0.993(0.985-1.001)$ & .083 \\
\hline & 6 & 1 & $1.004(0.996-1.011)$ & .275 & 1 & $0.995(0.987-1.003)$ & .229 \\
\hline & 7 & 1 & $1.006(0.998-1.013)$ & .057 & 1 & $0.995(0.987-1.003)$ & .212 \\
\hline \multirow[t]{8}{*}{$\mathrm{PM}_{10}\left(\mu \mathrm{g} / \mathrm{m}^{3}\right)$} & 0 & 7.12 & $1.005(1.002-1.008)$ & $<.001$ & 6.68 & $1.006(0.708-1.428)$ & .001 \\
\hline & 1 & 1.95 & $1.005(1.002-1.008)$ & $<.001$ & 2.54 & 1.005 (1.002-1.007) & .003 \\
\hline & 2 & 6.16 & $1.003(1.000-1.006)$ & .025 & 5.51 & $1.003(0.999-1.006)$ & .072 \\
\hline & 3 & 6.42 & 1.002 (0.999-1.005) & .142 & 2.35 & $1.002(0.999-1.005)$ & .145 \\
\hline & 4 & 6.68 & $1.002(0.998-1.005)$ & .146 & 1 & $1.001(0.997-1.004)$ & .562 \\
\hline & 5 & 7.35 & $1.000(0.997-1.004)$ & .587 & 7.22 & $1.003(0.999-1.006)$ & .059 \\
\hline & 6 & 6.68 & $1.002(0.999-1.005)$ & .146 & 6.17 & $1.002(0.999-1.006)$ & .071 \\
\hline & 7 & 6.68 & $1.002(0.999-1.005)$ & .111 & 6.15 & $1.002(0.999-1.005)$ & .132 \\
\hline
\end{tabular}

pooled results of studies analyzed by the GAM method showed that $\mathrm{PM}_{10}$ was associated with increased mortality from cardiovascular diseases, and for each $1 \mu \mathrm{g} /$ $\mathrm{m}^{3}$ increase in the concentration of $\mathrm{PM}_{10}$, cardiovascular death increased by $2.2 \%$ (Cl $95 \%$ : 1.6 to $2.8 \%$ ), but the pooled results of another study that did not use the GAM model, showed the increase in cardiovascular death was 1.3\% (Cl 95\%: 0.8-1.9\%) (Stieb et al. 2003). In a study done by Dastoorpoor et al. in Ahvaz, Iran; $10 \mu \mathrm{g} / \mathrm{m}^{3}$ increase in $\mathrm{PM}_{10}$, increased cardiovascular deaths by $1.012 \%$ (95\% Cl:1.001-1.023\%) (Dastoorpoor et al. 2018). In a study done by Middleton et al. in Nicosia, Cyprus, every $10 \mathrm{mg} / \mathrm{m}^{3}$ increase in $\mathrm{PM}_{10}$ concentration increased hospital admissions because of cardiovascular diseases by $1.2 \%(95 \% \mathrm{Cl}:-0.0 \%, 2.4 \%)$ (Middleton et al. 2008). In Vahedian et al.'s study conducted in Arak, Iran; for every $10 \mu \mathrm{g} / \mathrm{m}^{3}$ increase in $\mathrm{PM}_{10}$, hospital admissions for cardiovascular diseases increased $\quad 0.7 \% \quad(95 \% \quad \mathrm{Cl}: \quad 0.02-1.2 \%) \quad$ (Vahedian et al. 2017).

However, in some studies, the results were inconsistent with our results, for example in studies from Mashhad, Iran (Ghorbani et al. 2017), Shiraz, Iran (Dadbakhsh et al. 2016), and Kerman, Iran (Hashemi et al. 2014), $\mathrm{PM}_{10}$ did not have a significant direct relation with cardiovascular mortality; although, in these three studies the concentration of $\mathrm{PM}_{10}$ was more than the $\mathrm{PM}_{10}$ concentration in Tehran. One reason for these different results may be that negative binomial regression models were used in these previous studies, which are different and less advanced than the GAM models. Also the time unit in the Kerman (Hashemi et al. 2014) and Shiraz (Dadbakhsh et al. 2016) study was month, and in the Mashhad study (Ghorbani et al. 2017) no confounder variable was used in the model. 
Table 7. Results of Adjusted Generalized Additive Model, about the effect of air pollutants on female cardiovascular death, for 1 unit increase in $\mathrm{CO}$ and 10 units increase in all other pollutants (adjusted for relative humidity, temperature, trend, season and day of week).

\begin{tabular}{|c|c|c|c|c|c|c|c|}
\hline & \multirow[b]{2}{*}{ lag } & \multicolumn{3}{|c|}{ One pollutant } & \multicolumn{3}{|c|}{ Two pollutant } \\
\hline & & df & $\mathrm{RR}(95 \% \mathrm{Cl})$ & $p$ & df & $\mathrm{RR}(95 \% \mathrm{Cl})$ & $p$ \\
\hline \multirow[t]{8}{*}{$\mathrm{CO}$ (ppm) } & 0 & 1.04 & $0.989(0.978-1.000)$ & .051 & 1 & $0.992(0.981-1.003)$ & .177 \\
\hline & 1 & 1 & $0.992(0.981-1.003)$ & .16 & 1 & $0.997(0.986-1.008)$ & .58 \\
\hline & 2 & 2.84 & $0.989(0.979-1.001)$ & .068 & 2.07 & $0.992(0.982-1.003)$ & .174 \\
\hline & 3 & 3.34 & $0.991(0.981-1.002)$ & .111 & 1.68 & $0.992(0.981-1.003)$ & .141 \\
\hline & 4 & 4.27 & $0.994(0.983-1.005)$ & .231 & 1 & $0.989(0.982-1.004)$ & .179 \\
\hline & 5 & 6.16 & $0.993(0.983-1.004)$ & .236 & 6.38 & $0.998(0.987-1.009)$ & .753 \\
\hline & 6 & 1 & $0.998(0.987-1.009)$ & .782 & 1 & $1.001(0.990-1.012)$ & .844 \\
\hline & 7 & 1 & $1.001(0.989-1.011)$ & .923 & 1 & $1.001(0.990-1.012)$ & .826 \\
\hline \multirow[t]{8}{*}{$\mathrm{O}_{3}(\mathrm{ppb})$} & 0 & 2.37 & $1.015(1.005-1.024)$ & .002 & 2.11 & $1.012(1.003-1.022)$ & .012 \\
\hline & 1 & 2.69 & $1.016(1.007-1.026)$ & $<.001$ & 2.53 & $1.016(1.007-1.026)$ & .001 \\
\hline & 2 & 4.31 & $1.016(1.006-1.025)$ & $<.001$ & 1.57 & $1.015(1.005-1.024)$ & .002 \\
\hline & 3 & 1.59 & $1.013(1.004-1.023)$ & .005 & 1 & $1.013(1.004-1.023)$ & .005 \\
\hline & 4 & 1.25 & $1.003(0.994-1.013)$ & .078 & 1.2 & 1.012 (1.005-1.019) & .014 \\
\hline & 5 & 1.93 & $1.006(0.996-1.015)$ & .203 & 2.21 & $1.006(0.996-1.015)$ & .203 \\
\hline & 6 & 3.35 & $1.005(0.996-1.015)$ & .277 & 3.62 & $1.007(0.997-1.016)$ & .154 \\
\hline & 7 & 2.56 & $1.006(0.998-1.016)$ & .193 & 2.11 & $1.006(0.997-1.016)$ & .188 \\
\hline \multirow[t]{8}{*}{$\mathrm{NO}_{2}(\mathrm{ppb})$} & 0 & 1 & $1.002(0.997-1.008)$ & .38 & 1 & $0.999(0.994-1.005)$ & .858 \\
\hline & 1 & 1.01 & $1.004(0.998-1.009)$ & .142 & 3.68 & $1.000(0.995-1.006)$ & .927 \\
\hline & 2 & 2.73 & $1.003(0.997-1.008)$ & .269 & 2.75 & $0.998(0.993-1.004)$ & .526 \\
\hline & 3 & 3.55 & $1.004(0.999-1.009)$ & .111 & 3.79 & $0.999(0.994-1.005)$ & .981 \\
\hline & 4 & 1 & $1.003(0.997-1.008)$ & .238 & 4.15 & $1.003(0.997-1.008)$ & .33 \\
\hline & 5 & 3.95 & $1.005(0.999-1.010)$ & .065 & 4.27 & $1.006(1.000-1.011)$ & .034 \\
\hline & 6 & 1.10 & $1.006(1.001-1.011)$ & .023 & 1 & $0.999(0.994-1.005)$ & .916 \\
\hline & 7 & 1 & $1.003(0.998-1.008)$ & .207 & 1 & $1.004(0.998-1.009)$ & .182 \\
\hline \multirow[t]{8}{*}{$\mathrm{SO}_{2}(\mathrm{ppb})$} & 0 & 7.93 & $1.003(0.995-1.011)$ & .444 & 7.77 & $1.005(0.996-1.015)$ & .241 \\
\hline & 1 & 7.82 & $1.004(0.996-1.012)$ & .297 & 1 & $1.003(0.993-1.012)$ & .556 \\
\hline & 2 & 1 & $1.002(0.993-1.009)$ & .701 & 1 & $1.002(0.993-1.012)$ & .587 \\
\hline & 3 & 5.98 & $1.002(0.993-1.010)$ & .632 & 1 & $1.005(0.996-1.014)$ & .256 \\
\hline & 4 & 1 & $1.001(0.992-1.009)$ & .783 & 1 & $1.004(0.995-1.013)$ & .264 \\
\hline & 5 & 6.64 & $1.005(0.997-1.013)$ & .197 & 6.12 & 1.009 (0.999-1.018) & .056 \\
\hline & 6 & 7.53 & $1.005(0.997-1.014)$ & .182 & 7.84 & $1.003(0.994-1.012)$ & .401 \\
\hline & 7 & 8.09 & 1.007 (0.999-1.015) & .081 & 7.77 & $1.008(0.998-1.017)$ & .087 \\
\hline \multirow[t]{8}{*}{$\mathrm{PM}_{10}\left(\mu \mathrm{g} / \mathrm{m}^{3}\right)$} & 0 & 1.05 & $1.008(1.001-1.018)$ & $<.001$ & 1 & $1.006(1.003-1.010)$ & $<.001$ \\
\hline & 1 & 1 & 1.007 (1.003-1.010) & $<.001$ & 1 & $1.006(1.003-1.007)$ & $<.001$ \\
\hline & 2 & 6.46 & $1.005(1.001-1.008)$ & .008 & 6.6 & $1.004(1.001-1.008)$ & .011 \\
\hline & 3 & 3.90 & $1.003(0.999-1.006)$ & .094 & 4.39 & $1.003(0.999-1.006)$ & .126 \\
\hline & 4 & 3.37 & $1.003(0.999-1.006)$ & .083 & 2.48 & $1.001(0.997-1.004)$ & .66 \\
\hline & 5 & 5.18 & $1.001(0.997-1.004)$ & .576 & 5.35 & $1.001(0.997-1.004)$ & .444 \\
\hline & 6 & 3.34 & $1.002(0.998-1.005)$ & .269 & 4.35 & $1.002(0.998-1.005)$ & .302 \\
\hline & 7 & 3.34 & $1.002(0.999-1.006)$ & .173 & 1 & $1.025(0.986-1.055)$ & .242 \\
\hline
\end{tabular}

Ambient particulate matter may cause heart disease and cardiovascular death through increase in blood clotting, impaired heart function, increased blood viscosity and changes in heart rate (Qian et al. 2007).

The World Health Organization guidelines state that reduction of particulate matter $\left(\mathrm{PM}_{10}\right)$ from 70 to $20 \mu \mathrm{g} / \mathrm{m}^{3}$ can decrease air pollution-related deaths by around $15 \%$. In this study, the annual average of $\mathrm{PM}_{10}$ was higher than the WHO recommended 2014 guideline.

Another pollutant that showed a significant relation with cardiovascular deaths in this study was Nitrogen dioxide. $\mathrm{NO}_{2}$ was associated with all cardiovascular deaths, and with each $10 \mathrm{ppb}$ increase in $\mathrm{NO}_{2}$, total cardiovascular deaths increased $(0.88 \%, 95 \% \mathrm{Cl}: 0.3-0.99 \%)$ in lag 3 in the one pollutant; and $(0.57 \%, 95 \%$ Cl: $0.23-0.92 \%)$ in lag 1 , in two pollutant models. In this study, $\mathrm{NO}_{2}$ showed relations with cardiovascular death in men, women and elder age groups, as well. Ghorbani et al. study's in Mashhad, Iran, also showed that by $1 \mathrm{ppb}$ increase in $\mathrm{NO}_{2}$, all cardiovascular deaths increased by $1 \%$ (95\% Cl:0.6 to 1.4) (Ghorbani et al. 2017). In one study in 8 Chinese cities, each $10 \mu \mathrm{g} / \mathrm{m}^{3}$ increase in $\mathrm{NO}_{2}$, related to $1.3 \%(95 \% \mathrm{Cl}: 0.45-2.14)$ increase in coronary heart disease mortality after 2 days lag (Li et al. 2015). In a study from Shiraz NO $(\mathrm{RR}=1.00229, \quad 95 \% \mathrm{Cl}: 1.00031-1.00426)$ and $\mathrm{NO}_{\mathrm{x}}$ $(\mathrm{RR}=1.00187,95 \% \mathrm{Cl}: 1.00016-1.003)$ were related to cardiovascular disease mortality, but $\mathrm{NO}_{2}$ had no relation with cardiovascular deaths $(\mathrm{RR}=1.00429,95 \% \mathrm{Cl}$ : 0.99637-1.01228) (Dadbakhsh et al. 2016).

$\mathrm{NO}_{2}$ is a gas with a red-orange (almost brown) color. It has a boiling point of $21.2^{\circ} \mathrm{C}$. The toxicity of $\mathrm{NO}_{2}$ is several times higher than $\mathrm{NO}$ in humans. $\mathrm{NO}_{2}$ 
Table 8. Results of Adjusted Generalized Additive Model, about the effect of air pollutants on under 18 years old cardiovascular death, for 1 unit increase in $\mathrm{CO}$ and 10 units increase in all other pollutants (adjusted for relative humidity, temperature, trend, season and day of week).

\begin{tabular}{|c|c|c|c|c|c|c|c|}
\hline & \multirow[b]{2}{*}{ lag } & \multicolumn{3}{|c|}{ One pollutant } & \multicolumn{3}{|c|}{ Two pollutant } \\
\hline & & $\mathrm{df}$ & RR $(95 \% \mathrm{Cl})$ & $p$ & df & RR $(95 \% \mathrm{Cl})$ & $p$ \\
\hline \multirow{8}{*}{$\mathrm{CO}(\mathrm{ppm})$} & 0 & 1 & $1.013(0.957-1.072)$ & .654 & 1.31 & $0.946(0.827-1.083)$ & .424 \\
\hline & 1 & 1.35 & $1.027(0.971-1.086)$ & .357 & 1.49 & $0.991(0.866-1.133)$ & .891 \\
\hline & 2 & 1 & $1.009(0.953-1.069)$ & .744 & 1 & $0.962(0.838-1.104)$ & .583 \\
\hline & 3 & 1 & $1.027(0.971-1.088)$ & .341 & 1.49 & $0.926(0.803-1.067)$ & .291 \\
\hline & 4 & 1 & $1.021(0.969-1.076)$ & .404 & 1.12 & $0.959(0.837-1.099)$ & .551 \\
\hline & 5 & 1 & $1.039(0.982-1.100)$ & .175 & 2.21 & $0.936(0.817-1.072)$ & .340 \\
\hline & 6 & 1 & $1.049(0.992-1.109)$ & .059 & 1 & $0.951(0.832-1.087)$ & .465 \\
\hline & 7 & 1 & $1.014(0.958-1.074)$ & .623 & 1 & $1.072(0.934-1.231)$ & .322 \\
\hline \multirow{8}{*}{$\mathrm{O}_{3}(\mathrm{ppb})$} & 0 & 5.75 & $0.985(0.937-1.036)$ & .564 & 5.96 & $1.072(0.959-1.197)$ & .221 \\
\hline & 1 & 1.33 & $0.995(0.949-1.044)$ & .854 & 1 & $1.081(0.976-1.198)$ & .134 \\
\hline & 2 & 1 & $0.971(0.923-1.022)$ & .254 & 1 & $1.021(0.917-1.136)$ & .702 \\
\hline & 3 & 1.36 & $0.967(0.921-1.015)$ & .179 & 1 & $0.966(0.870-1.073)$ & .618 \\
\hline & 4 & 1 & $0.969(0.923-1.018)$ & .215 & 1 & $0.976(0.879-1.084)$ & .492 \\
\hline & 5 & 7.49 & $0.969(0.921-1.019)$ & .229 & 7.34 & $0.924(0.828-1.030)$ & .154 \\
\hline & 6 & 4.65 & $0.981(0.931-1.033)$ & .464 & 1 & $1.019(0.915-1.134)$ & .735 \\
\hline & 7 & 1 & $0.981(0.932-1.031)$ & .445 & 4.5 & $1.030(0.927-1.144)$ & .576 \\
\hline \multirow{8}{*}{$\mathrm{NO}_{2}(\mathrm{ppb})$} & 0 & 1 & $1.011(0.983-1.039)$ & .428 & 1 & $1.023(0.955-1.096)$ & .505 \\
\hline & 1 & 1.01 & $1.026(0.998-1.054)$ & .068 & 1 & $1.015(0.951-1.083)$ & .429 \\
\hline & 2 & 1 & $1.011(0.983-1.039)$ & .44 & 1 & $0.997(0.931-1.068)$ & .942 \\
\hline & 3 & 2.57 & $1.014(0.986-1.043)$ & .314 & 1 & $1.022(0.954-1.093)$ & .537 \\
\hline & 4 & 2.02 & $1.004(0.977-1.032)$ & .321 & 1.6 & $1.021(0.958-1.089)$ & .295 \\
\hline & 5 & 2.07 & $1.007(0.979-1.035)$ & .625 & 1.13 & $1.128(0.996-1.278)$ & .342 \\
\hline & 6 & 1 & $1.015(0.987-1.044)$ & .284 & 1.08 & $0.997(0.927-1.065)$ & .857 \\
\hline & 7 & 1.46 & $1.007(0.979-1.035)$ & .628 & 1 & $1.065(0.996-1.139)$ & .063 \\
\hline \multirow{8}{*}{$\mathrm{SO}_{2}(\mathrm{ppb})$} & 0 & 1.67 & $1.029(0.987-1.074)$ & .172 & 1.82 & $0.947(0.826-1.085)$ & .434 \\
\hline & 1 & 1.20 & $1.041(0.998-1.085)$ & .061 & 1.83 & $1.126(0.989-1.282)$ & .071 \\
\hline & 2 & 1.02 & $1.055(0.998-1.114)$ & .163 & 1.1 & $1.058(0.929-1.204)$ & .086 \\
\hline & 3 & 1.01 & $1.049(0.982-1.123)$ & .219 & 1 & $1.023(0.896-1.168)$ & .736 \\
\hline & 4 & 2.14 & $1.061(0.978-1.149)$ & .228 & 7.55 & $1.012(0.887-1.156)$ & .849 \\
\hline & 5 & 2.23 & $1.027(0.946-1.114)$ & .312 & 1 & $1.005(0.882-1.145)$ & .943 \\
\hline & 6 & 1.05 & $1.076(0.980-1.181)$ & .621 & 1 & $1.035(0.908-1.179)$ & .871 \\
\hline & 7 & 1 & $1.038(0.955-1.128)$ & .369 & 1.06 & $0.997(0.874-1.137)$ & .964 \\
\hline \multirow[t]{8}{*}{$\mathrm{PM}_{10}\left(\mu \mathrm{g} / \mathrm{m}^{3}\right)$} & 0 & 1 & $0.994(0.975-1.012)$ & .508 & 1 & $0.994(0.956-1.034)$ & .782 \\
\hline & 1 & 1 & $0.999(0.981-1.017)$ & .916 & 1 & $0.985(0.946-1.025)$ & .461 \\
\hline & 2 & 1 & $1.000(0.982-1.019)$ & .965 & 2.06 & $1.013(0.974-1.052)$ & .520 \\
\hline & 3 & 1.01 & $0.994(0.976-1.013)$ & .543 & 1 & $0.996(0.958-1.035)$ & .829 \\
\hline & 4 & 1 & $0.995(0.973-1.017)$ & .691 & 1 & $0.995(0.961-1.031)$ & .618 \\
\hline & 5 & 1 & $1.002(0.985-1.021)$ & .785 & 1 & $1.014(0.976-1.053)$ & .467 \\
\hline & 6 & 1 & $1.003(0.985-1.021)$ & .744 & 1 & $1.012(0.975-1.051)$ & .511 \\
\hline & 7 & 1 & $1.011(0.993-1.029)$ & .236 & 1 & $0.995(0.959-1.032)$ & .813 \\
\hline
\end{tabular}

at 15 ppm concentration can damage human kidney, liver and heart tissues after just $2 \mathrm{~h}$ contact (Bahrami Asl et al. 2014). The main mechanism of toxicity of $\mathrm{NO}_{2}$ is intervening with the peroxidation of lipids in cell membranes. Its free radicals have various adverse effects on structural and functional molecules (Kelly et al. 1996). According to the World Health Organization's guideline the mean annual threshold for $\mathrm{NO}_{2}$ is $40 \mu \mathrm{g} / \mathrm{m}^{3}$.

In this study, sulfur dioxide showed a significant relation with cardiovascular deaths in the one pollutant model, and for $10 \mathrm{ppb}$ increase in $\mathrm{SO}_{2}$, total death increased by $0.89 \%$ (95\% $\mathrm{Cl}: 0.36-1.62 \%)$. According to a study done by Hong et al. in Korea, sulfur dioxide showed a relation with ischemic stroke as well; and the Rate Ratio was 1.04 (95\% Cl, 1.01 to 1.08) (Hong et al. 2002). In a study from Shiraz, a relation was observed between $\mathrm{SO}_{2}$ and cardiovascular mortality in women, and the IRR was $1.00089 \quad(95 \% \mathrm{Cl}$ : 1.00008-1.00171) for each $1 \mathrm{ppb}$ increase in $\mathrm{SO}_{2}$ (Dadbakhsh et al. 2016). In a study done in Brazil, the 7-day moving average of $\mathrm{SO}_{2}$ was significantly related with mortality due to circulatory diseases and the RR was $1.04(95 \% \mathrm{Cl}=1.01-1.06)$, after adjusting for ozone (Amancio and Nascimento, 2012). Zeng et al showed that $\mathrm{SO}_{2}$ per $10 \mu \mathrm{g} / \mathrm{m}^{3}$ increase and after one day lag, increased cardiovascular deaths by $0.48 \%(95 \% \mathrm{Cl}$ : 0.11-0.85\%) (Zeng et al. 2015).

Researchers think sulfur dioxide exacerbates cardiovascular complications and causes death. But many questions about the effects of sulfur dioxide on human health remain unanswered. Sulfur oxides 
Table 9. Results of Adjusted Generalized Additive Model, about the effect of air pollutants on 18 to 60 years old cardiovascular death, for 1 unit increase in $\mathrm{CO}$ and 10 units increase in all other pollutants (Adjusted for relative humidity, temperature, trend, season and day of week).

\begin{tabular}{|c|c|c|c|c|c|c|c|}
\hline & \multirow[b]{2}{*}{ lag } & \multicolumn{3}{|c|}{ One pollutant } & \multicolumn{3}{|c|}{ Two pollutant } \\
\hline & & df & RR $(95 \% \mathrm{Cl})$ & $p$ & df & RR $(95 \% \mathrm{Cl})$ & $p$ \\
\hline \multirow{8}{*}{ CO (ppm) } & 0 & 7.3 & $0.997(0.985-1.010)$ & .666 & 7.45 & $0.996(0.983-1.008)$ & .49 \\
\hline & 1 & 1.7 & $1.002(0.989-1.014)$ & .783 & 2.12 & $1.001(0.988-1.014)$ & .857 \\
\hline & 2 & 2.44 & $0.998(0.986-1.011)$ & .802 & 3.3 & $0.997(0.985-1.010)$ & .727 \\
\hline & 3 & 5.88 & $0.996(0.983-1.008)$ & .509 & 6.15 & $0.994(0.982-1.007)$ & .391 \\
\hline & 4 & 6.76 & $0.997(0.983-1.010)$ & .526 & 7.2 & $0.992(0.979-1.005)$ & .208 \\
\hline & 5 & 7.37 & $0.995(0.982-1.007)$ & .433 & 7.6 & $0.991(0.978-1.003)$ & .139 \\
\hline & 6 & 1 & $0.993(0.981-1.006)$ & .286 & 2.29 & $0.991(0.980-1.002)$ & .096 \\
\hline & 7 & 2.51 & $0.995(0.983-1.008)$ & .471 & 4.78 & $0.993(0.980-1.005)$ & .255 \\
\hline \multirow{8}{*}{$\mathrm{O}_{3}(\mathrm{ppb})$} & 0 & 7.73 & $0.995(0.984-1.006)$ & .382 & 7.81 & $0.997(0.986-1.008)$ & .658 \\
\hline & 1 & 3.20 & $0.999(0.988-1.009)$ & .855 & 3 & $1.002(0.991-1.013)$ & .729 \\
\hline & 2 & 3.04 & $0.999(0.988-1.010)$ & .858 & 2.53 & $1.002(0.991-1.012)$ & .785 \\
\hline & 3 & 3.21 & $0.997(0.987-1.008)$ & .675 & 2.77 & $1.001(0.989-1.012)$ & .916 \\
\hline & 4 & 3.78 & $0.998(0.986-1.011)$ & .375 & 3.54 & $0.993(0.982-1.004)$ & .232 \\
\hline & 5 & 4.34 & $0.990(0.979-1.001)$ & .077 & 4.22 & $0.994(0.984-1.005)$ & .316 \\
\hline & 6 & 5.35 & $0.991(0.980-1.002)$ & .099 & 4.12 & $0.996(0.985-1.007)$ & .448 \\
\hline & 7 & 4.49 & $0.992(0.982-1.003)$ & .177 & 5.34 & $0.996(0.985-1.007)$ & .457 \\
\hline \multirow[t]{8}{*}{$\mathrm{NO}_{2}(\mathrm{ppb})$} & 0 & 2.04 & $1.007(1.001-1.013)$ & .019 & 1.95 & $1.008(1.000-1.016)$ & .044 \\
\hline & 1 & 1 & $1.008(1.002-1.014)$ & .01 & 1 & $1.008(1.001-1.016)$ & .035 \\
\hline & 2 & 1 & $1.007(1.001-1.013)$ & .03 & 1 & $1.005(1.001-1.013)$ & .026 \\
\hline & 3 & 1 & 1.007 (1.001-1.013) & .019 & 1 & $1.013(1.005-1.021)$ & .002 \\
\hline & 4 & 1.07 & $1.006(0.999-1.013)$ & .152 & 4.65 & $1.092(1.001-1.017)$ & .020 \\
\hline & 5 & 1.74 & 1.007 (0.999-1.014) & .128 & 3.5 & $1.009(1.002-1.017)$ & .016 \\
\hline & 6 & 1.19 & $1.007(0.999-1.014)$ & .216 & 1 & $1.008(0.999-1.015)$ & .051 \\
\hline & 7 & 1 & $1.006(0.999-1.012)$ & .193 & 1 & $1.006(0.998-1.014)$ & .109 \\
\hline \multirow{8}{*}{$\mathrm{SO}_{2}(\mathrm{ppb})$} & 0 & 8.45 & $1.009(1.000-1.019)$ & .04 & 8.12 & $1.002(0.989-1.014)$ & .769 \\
\hline & 1 & 8.37 & $1.008(0.998-1.017)$ & .099 & 1 & $0.998(0.983-1.008)$ & .512 \\
\hline & 2 & 8.33 & $1.005(0.996-1.015)$ & .249 & 8.02 & $0.996(0.983-1.008)$ & .505 \\
\hline & 3 & 8.24 & $1.005(0.995-1.014)$ & .267 & 1 & $0.998(0.985-1.011)$ & .771 \\
\hline & 4 & 1 & $1.004(0.994-1.014)$ & .333 & 1 & $0.997(0.985-1.009)$ & .434 \\
\hline & 5 & 1 & $1.006(0.997-1.015)$ & .195 & 1 & $1.000(0.988-1.013)$ & .957 \\
\hline & 6 & 1 & $1.005(0.995-1.014)$ & .325 & 7.39 & $0.996(0.983-1.008)$ & .521 \\
\hline & 7 & 1 & $1.007(0.997-1.016)$ & .14 & 1 & $1.001(0.988-1.016)$ & .858 \\
\hline \multirow[t]{8}{*}{$\mathrm{PM}_{10}\left(\mu \mathrm{g} / \mathrm{m}^{3}\right)$} & 0 & 1.99 & $1.006(1.002-1.010)$ & .004 & 2.78 & 1.005 (1.001-1.009) & .007 \\
\hline & 1 & 1.96 & $1.005(1.002-1.009)$ & .004 & 2.44 & $1.005(1.001-1.009)$ & .007 \\
\hline & 2 & 7.48 & $1.004(0.999-1.007)$ & .082 & 7.49 & $1.003(0.999-1.007)$ & .101 \\
\hline & 3 & 6.95 & $1.002(0.998-1.006)$ & .223 & 6.6 & $1.002(0.998-1.006)$ & .281 \\
\hline & 4 & 6.34 & $1.001(0.997-1.005)$ & .264 & 5.37 & $1.000(0.996-1.004)$ & .834 \\
\hline & 5 & 7.07 & $1.001(0.997-1.005)$ & .601 & 6.77 & $1.002(0.997-1.005)$ & .491 \\
\hline & 6 & 6.79 & $1.002(0.998-1.006)$ & .298 & 6.36 & $1.002(0.998-1.006)$ & .322 \\
\hline & 7 & 7.78 & $1.002(0.998-1.006)$ & .252 & 6.27 & $1.018(0.978-1.059)$ & .378 \\
\hline
\end{tabular}

tends to be present in polluted air containing suspended solids and severe moisture and as a result, few epidemiologic studies are able to distinguish the effects of these pollutants separately (Goudarzi et al. 2014).

In this study, $\mathrm{O}_{3}$ showed no significant relation with cardiovascular deaths in males and in total. In some other studies, similar results have been seen (Wong et al. 2002, Hashemi et al. 2014, Dadbakhsh et al. 2016, Ghorbani et al. 2017). But in women, after a 1 day lag, with $10 \mathrm{ppb}$ increase of $\mathrm{O}_{3}$, cardiovascular deaths increased $1.66 \%(95 \% \mathrm{Cl}: 0.73-2.61 \%)$ in the one pollutant and $1.65 \%(0.71-2.61 \%)$ in the two pollutant model. Some other studies have shown a relation between $\mathrm{O}_{3}$ with cardiovascular death as well. Zhang et al. showed that for each $10 \mu \mathrm{g} / \mathrm{m}^{3}$ increase in $\mathrm{O}_{3}$ concentration, cardiovascular deaths increased by 0.45\% (Cl 95\%: 0.16-0.73) in Shanghai (Zhang et al. 2006). In a meta-analysis done in 2005, the short-term effects of exposure to ozone and cardiovascular and respiratory mortality were analyzed. The pooled results showed that a 10 ppb increase in daily ozone, in lags 0 , 1 , or 2 days was associated with a $0.87 \% \quad(95 \%$ $\mathrm{Cl}=0.55-1.18$ ) increase in overall (male and female) mortality. But in some studies ozone had no significant relation with cardiovascular deaths (Dadbakhsh et al. 2016). Kan et al. reported that the effect of $\mathrm{O}_{3}$ on total mortality in females was higher than males; and gender was an effect modifier in Shanghai, China (Kan et al. 2008). However, the mechanism of effect of ozone on cardiovascular deaths is still not known and requires further investigation (Ghanbari Ghozikali et al. 2014). 
Table 10. Results of Adjusted Generalized Additive Model, about the effect of air pollutants on over 60 years old cardiovascular death, for 1 unit increase in $\mathrm{CO}$ and 10 units increase in all other pollutants (Adjusted for relative humidity, temperature, trend, season and day of week).

\begin{tabular}{|c|c|c|c|c|c|c|c|}
\hline & \multirow[b]{2}{*}{ lag } & \multicolumn{3}{|c|}{ One pollutant } & \multicolumn{3}{|c|}{ Two pollutant } \\
\hline & & df & $\mathrm{RR}(95 \% \mathrm{Cl})$ & $p$ & df & $\mathrm{RR}(95 \% \mathrm{Cl})$ & $p$ \\
\hline \multirow[t]{8}{*}{ CO (ppm) } & 0 & 7.17 & $0.998(0.989-1.007)$ & .663 & 2.28 & $0.996(0.987-1.005)$ & .388 \\
\hline & 1 & 1 & $1.002(0.993-1.011)$ & .623 & 1.89 & $1.002(0.993-1.011)$ & .709 \\
\hline & 2 & 2.54 & $0.998(0.989-1.007)$ & .738 & 4.32 & $0.998(0.989-1.007)$ & .653 \\
\hline & 3 & 5.47 & $0.995(0.987-1.004)$ & .319 & 5.83 & $0.994(0.985-1.003)$ & .211 \\
\hline & 4 & 6.41 & $0.995(0.985-1.006)$ & .461 & 7 & $0.991(0.983-1.000)$ & .058 \\
\hline & 5 & 7.03 & $0.994(0.986-1.003)$ & .273 & 7.42 & $0.989(0.981-0.999)$ & .023 \\
\hline & 6 & 4.1 & $0.992(0.984-1.001)$ & .091 & 1 & $0.994(0.984-1.002)$ & .149 \\
\hline & 7 & 1 & $0.995(0.986-1.004)$ & .303 & 1 & $0.992(0.984-0.999)$ & .031 \\
\hline \multirow[t]{8}{*}{$\mathrm{O}_{3}(\mathrm{ppb})$} & 0 & 7.86 & $0.996(0.988-1.003)$ & .315 & 7.92 & $0.998(0.991-1.006)$ & 672 \\
\hline & 1 & 3.2 & $1.000(0.992-1.007)$ & .997 & 2.72 & $1.003(0.995-1.010)$ & .475 \\
\hline & 2 & 3.24 & $0.999(0.992-1.008)$ & .846 & 2.54 & $1.001(0.994-1.000)$ & .711 \\
\hline & 3 & 3.48 & $0.998(0.991-1.005)$ & .614 & 2.84 & $1.001(0.993-1.008)$ & .863 \\
\hline & 4 & 3.82 & $0.999(0.992-1.007)$ & .546 & 3.55 & $0.993(0.986-1.001)$ & .089 \\
\hline & 5 & 4.37 & $0.990(0.983-0.998)$ & .016 & 4.24 & $0.995(0.987-1.002)$ & .175 \\
\hline & 6 & 5.46 & 0.997 (0.984-0.999) & .029 & 4.22 & $0.995(0.988-1.003)$ & .25 \\
\hline & 7 & 4.46 & $0.993(0.985-1.000)$ & .056 & 5.38 & $0.996(0.988-1.003)$ & .288 \\
\hline \multirow[t]{8}{*}{$\mathrm{NO}_{2}(\mathrm{ppb})$} & 0 & 2.54 & $1.006(1.002-1.011)$ & .002 & 1.7 & $1.006(1.001-1.011)$ & .031 \\
\hline & 1 & 1.29 & 1.007 (1.003-1.012) & $<.001$ & 1 & $1.012(1.006-1.017)$ & $<.001$ \\
\hline & 2 & 1 & $1.006(1.002-1.010)$ & .005 & 1 & $1.007(1.001-1.012)$ & .012 \\
\hline & 3 & 1 & $1.006(1.002-1.011)$ & .002 & 1 & $1.007(1.002-1.013)$ & .007 \\
\hline & 4 & 4.17 & $1.004(1.000-1.008)$ & .001 & 4.54 & 1.006 (1.003-1.009) & $<.001$ \\
\hline & 5 & 1.96 & $1.007(1.003-1.012)$ & $<.001$ & 3.25 & 1.009 (1.004-1.015) & $<.001$ \\
\hline & 6 & 1.49 & $1.008(1.004-1.013)$ & $<.001$ & 1 & $1.008(1.002-1.013)$ & .005 \\
\hline & 7 & 1 & $1.008(1.004-1.012)$ & .002 & 1 & $1.006(0.996-1.015)$ & .176 \\
\hline \multirow[t]{8}{*}{$\mathrm{SO}_{2}(\mathrm{ppb})$} & 0 & 8.43 & 1.009 (1.003-1.015) & .006 & 8.18 & $1.002(0.993-1.010)$ & .731 \\
\hline & 1 & 1 & $1.007(1.001-1.013)$ & .033 & 1 & $0.996(0.987-1.005)$ & .373 \\
\hline & 2 & 8.25 & $1.005(0.998-1.011)$ & .131 & 7.9 & $0.997(0.988-1.005)$ & .452 \\
\hline & 3 & 1 & $1.005(0.998-1.011)$ & .139 & 1 & $0.999(0.994-1.004)$ & .226 \\
\hline & 4 & 1 & $1.003(0.995-1.009)$ & .377 & 1 & $0.999(0.990-1.007)$ & .825 \\
\hline & 5 & 7.84 & $1.005(0.998-1.012)$ & .11 & 1 & $1.000(0.992-1.009)$ & .923 \\
\hline & 6 & 1.01 & $1.004(0.997-1.010)$ & .234 & 7.39 & $0.996(0.987-1.005)$ & .384 \\
\hline & 7 & 7.84 & $1.006(0.999-1.013)$ & .059 & 7.38 & $1.001(0.992-1.009)$ & .871 \\
\hline \multirow{8}{*}{$\mathrm{PM}_{10}\left(\mu \mathrm{g} / \mathrm{m}^{3}\right)$} & 0 & 1.84 & $1.005(1.003-1.008)$ & .001 & 2.17 & $1.005(1.002-1.008)$ & $<.001$ \\
\hline & 1 & 1.57 & $1.005(1.0025-1.008)$ & .001 & 1 & $1.005(1.002-1.008)$ & $<.001$ \\
\hline & 2 & 7.49 & $1.003(1.001-1.006)$ & .017 & 7.48 & $1.003(1.000-1.006)$ & .023 \\
\hline & 3 & 6.9 & $1.002(0.999-1.005)$ & .138 & 6.52 & $1.000(0.997-1.003)$ & .198 \\
\hline & 4 & 6.21 & $1.001(0.996-1.006)$ & .124 & 1.98 & $1.004(0.998-1.009)$ & .154 \\
\hline & 5 & 6.94 & $1.001(0.997-1.003)$ & .658 & 6.63 & $1.001(0.998-1.004)$ & .509 \\
\hline & 6 & 6.81 & $1.002(0.999-1.004)$ & .196 & 6.45 & $1.002(0.999-1.005)$ & .063 \\
\hline & 7 & 6.82 & $1.002(0.999-1.005)$ & .149 & 6.32 & $1.002(0.999-1.005)$ & .054 \\
\hline
\end{tabular}

CO was not related with cardiovascular deaths in this study, and Dastoorpoor et al. did not see a relation between $\mathrm{CO}$ and cardiovascular deaths in Ahwaz either (Dastoorpoor et al. 2018). But some studies have shown a relation between this pollutant and cardiovascular deaths. In Hong et al.'s study in Korea, carbon monoxide was related to ischemic stroke and the Rate Ratio was 1.04 (95\% Cl=1.01-1.07) (Hong et al. 2002). One of the reasons for these controversial results may be that in our study and in the Ahvaz study, all cardiovascular deaths have been included, but Hong et al only included deaths from ischemic stroke. More investigation is needed about the effects of air pollutants on specific cardiovascular diseases.

In this present study, $\mathrm{NO}_{2}, \mathrm{SO}_{2}$ and $\mathrm{PM}_{10}$ in people over 60 years had a stronger relation with cardiovascular deaths than the younger age group. This finding shows the stronger effects of $\mathrm{NO}_{2}, \mathrm{SO}_{2}$ and $\mathrm{PM}_{10}$ pollutants on the elderly people. Maheswaran et al, in south London also showed a higher risk of ischemic stroke in the 65 to 79-year old age group, and the RR for $\mathrm{NO}_{2}$ and $\mathrm{PM}_{10}$ were respectively $1.86(95 \% \mathrm{Cl}$ : 1.10-3.13) and 1.23 (95\% Cl: 0.99-1.53) (Maheswaran et al. 2012). The elderly are probably more susceptible to air pollution due to the consequences of natural aging (Bentayeb et al. 2012).

In some cases air pollutants may show significant negative relations with cardiovascular deaths. But this is usually due to the harvesting effect. In this study the RR for the effect of pollutants on cardiovascular deaths in percentiles above the $95^{\text {th }}$ percentile were compared to the $5^{\text {th }}$ percentile. Result showed that the ratio of count of deaths in the above the $95^{\text {th }}$ percentile range on the count of deaths in the under the $5^{\text {th }}$ percentile range were greater than 1 . And this shows that the harvesting effect has probably 
happened. Spix et al. have introduced methods for identifying the harvesting effect (Spix et al. 1993).

Ambient air pollutants may cause cardiovascular death through numerous mechanisms, such as accelerated atherosclerosis, changes in heart function, increased inflammatory cytokines in the heart, increased blood clotting, increased blood viscosity, increased plasma fibrinogen and changes in heart rate, lung inflammation and aggravation of lung disease (Brook et al. 2010).

Several studies have shown that ambient air pollutants are related to cardiovascular deaths. But, the different results between studies may be related to differences in the concentration of pollutants in different parts of the world, the statistical models use for data analysis, and adjusting for different confounders.

Mobile and stationary sources are both responsible for the air pollution crisis in Tehran. Motor vehicles are the main source for especially $\mathrm{CO}, \mathrm{NO}_{2}$ and $\mathrm{PM}_{10}$ in Tehran. Tehran also has a lot of factories and is an industrial hub. According to Mazaheri et al, mobile sources of air pollution are more important than stationary sources in making and emitting $\mathrm{NO}_{2}$ and $\mathrm{CO}$ in Tehran (Mazaheri Tehrani et al. 2015). Therefore, efforts to reduce pollutants released from mobile resources are essential.

\section{Strengths and limitations}

The strength of this study was that air pollution and cardiovascular death data from a ten-year period was used; and air pollution and meteorological data were obtained from reliable sources. Also as studies have shown that meteorological data, do not have a linear relation with health outcomes (Bhaskaran et al. 2009), in this study, we used Generalized Additive Models (GAM) for nonlinear confounder variables.

A limitation of this study was that about $8 \%$ of the air pollution data was missing. However, they were estimated using the EM algorithm method. Analysis was done separately for completed cases and imputed cases and the results were almost the same. But, in most cases analysis by EM imputed data produced higher precision.

Another limitation was that the impact of other potential confounding variables such as the concentrations of other pollutants, and wind direction were not investigated. We did not do variable interactions in this study either, but it can be an interesting topic for future researchers.

\section{Conclusion}

The results of this study showed that air pollution in Tehran may be responsible for some part of the cardiovascular deaths that happen in this city. Further efforts to control air pollutants, especially $\mathrm{PM}_{10}$, and $\mathrm{NO}_{2}$ in Tehran are essential. These efforts can include reducing emissions in the industrial and transport sector and strict regulations for using low sulfur gasoline or diesel filters. It is also recommended that elderly people with cardiovascular diseases avoid outdoor work and activity and use approved masks on days when air pollution is higher than the WHO standard guidelines.

\section{Acknowledgements}

The authors appreciate the Tehran Air Quality Control Company (AQCC), Tehran Department of Environmental Protection, Tehran Meteorological Organization and the Tehran Cemetery (Behesht-e-Zahra) for providing the data of air pollutants, meteorology indices and deaths. This article is part of a $\mathrm{PhD}$ thesis in Epidemiology done at Kerman University of Medical Sciences.

\section{Ethical approval}

This project was approved by the Standing Committee of Ethics in Research of Kerman University of Medical Sciences (Ethics code: IR.KMU.REC.1395.267).

\section{Disclosure statement}

No potential conflict of interest was reported by the authors.

\section{Funding}

This study was supported by Grant No. 95-198, from Kerman University of Medical Sciences, Kerman, Iran.

\section{References}

Afshari Safavi, A., Kazemzadeh Gharechobogh, H., and Rezaei, M., 2015. Comparison of EM algorithm and standard imputation methods for missing data: a questionnaire study on diabetic patients. Iranian journal of epidemiology, 11, 43-51.

Amancio, C., and Nascimento, L., 2012. Association of sulfur dioxide exposure with circulatory system deaths in a medium-sized city in Brazil. Brazilian journal of medical and biological research, 45 (11), 1080-1085.

Anava, O., Hazan, E. \& Zeevi, A. Online time series prediction with missing data. Proceedings of the 32nd International Conference on Machine Learning (ICML-15), 2015. 2191-2199. 
Bahrami Asl, F., et al., 2014. Estimation of diseases and mortality attributed to $\mathrm{NO}_{2}$ pollutant in five metropolises of Iran using AirQ model in 2011-2012. Journal of Mazandaran university of medical sciences, 24, 239-249.

Bentayeb, M., Geriatric Study in Europe on Health Effects of Air Quality in Nursing Homes Group, et al., 2012. Adverse respiratory effects of outdoor air pollution in the elderly. The international journal of tuberculosis and lung disease, 16 (9), 1149-1161.

Bhaskaran, K., et al., 2009. Effects of ambient temperature on the incidence of myocardial infarction - Systematic review. Heart, 95 (21), 1760-1769.

Brook, R.D., et al., 2010. Particulate matter air pollution and cardiovascular disease. Circulation, 121 (21), 2331-2378.

Dadbakhsh, M., Khanjani, N., and Bahrampour, A., 2016. Death from cardiovascular diseases and air pollution in Shiraz, Iran (March 2006-March 2012). Journal of epidemiology and preventive medicine, 02 (01), 114.

Dadbakhsh, M., Khanjani, N., and Bahrampour, A., 2018. The relation between mortality from cardiovascular diseases and temperature in Shiraz, Iran, 2006-2012. ARYA atherosclerosis, 14, 149.

Dastoorpoor, M., et al., 2018. Lag time structure of cardiovascular deaths attributed to ambient air pollutants in Ahvaz, Iran, 2008-2015. International journal of occupational medicine and environmental health, 31, 459-473.

Dastoorpoor, M., et al., 2019. Air pollution and hospital admissions for cardiovascular diseases in Ahvaz, Iran. Science of the total environment, 652, 1318-1330.

Dehghan, A., et al., 2018. The relation between air pollution and respiratory deaths in Tehran, Iran-using generalized additive models. BMC pulmonary medicine, 18 (1), 49.

Dempster, A.P., Laird, N.M., and Rubin, D.B., 1977. Maximum likelihood from incomplete data via the EM algorithm. Journal of the royal statistical society. series $B$ (methodological), 39 (1), 1-22.

Dominici, F., et al., 2002. On the use of generalized additive models in time-series studies of air pollution and health. American journal of epidemiology, 156 (3), 193-203.

Ghanbari Ghozikali, M., Mosaferi, M., and Nadafi, K., 2014. Quantification of the health effects of exposure to ozone in Tabriz by using AIRQ Model. Urmia medical journal, 25, 521-530.

Ghorbani, N., Yazdani Charati, J., and Etemadinejad, S., 2017. Relationship between air pollution and mortality rate due to cardiovascular diseases in Mashhad, Iran 2011. Journal of Mazandaran university of medical sciences, 26, 47-55.

Goudarzi, G., et al., 2014. Cardiovascular deaths related to carbon monoxide exposure in Ahvaz, Iran. Iranian journal of health, safety and environment, 1, 126-131.

Guisan, A., Edwards, T.C., and Hastie, T., 2002. Generalized linear and generalized additive models in studies of species distributions: setting the scene. Ecological modelling, 157 (2-3), 89-100.

Hashemi, S.Y., and Khanjani, N., 2016. Air pollution and cardiovascular hospital admissions in Kerman, Iran. Journal of Heart and Cardiology, 2 (2), 1-6.

Hashemi, S.Y., et al., 2014. Air pollution and cardiovascular mortality in Kerman from 2006 to 2011. American journal of cardiovascular disease research, 2, 27-30.
Hong, Y.-C., et al., 2002. Air pollution: a new risk factor in ischemic stroke mortality. Stroke, 33 (9), 2165-2169.

Hosseinpoor, A.R., et al., 2005. Air pollution and hospitalization due to angina pectoris in Tehran, Iran: a time-series study. Environmental research, 99 (1), 126-131.

Kan, H., et al., 2008. Season, sex, age, and education as modifiers of the effects of outdoor air pollution on daily mortality in Shanghai, China: The Public Health and Air Pollution in Asia (PAPA) Study. Environmental health perspectives, 116 (9), 1183.

Kelly, F.J., et al., 1996. Antioxidant kinetics in lung lavage fluid following exposure of humans to nitrogen dioxide. American journal of respiratory and critical care medicine, 154 (6), 1700-1705.

Khalilzadeh, S., et al., 2009. The relation between air pollution and cardiorespiratory admissions in Tehran. Tanaffos, 8, 35-40.

Khanjani, N., and Bahrampour, A., 2013. Temperature and cardiovascular and respiratory mortality in desert climate. A case study of Kerman, Iran. Iranian journal of environmental health science and engineering, 10, 11.

Li, H., et al., 2015. Short-term exposure to ambient air pollution and coronary heart disease mortality in 8 Chinese cities. International journal of cardiology, 197, 265-270.

López-Villarrubia, E., et al., 2010. Air pollution and mortality in the Canary Islands: a time-series analysis. Environmental Health, 9, 8

Maheswaran, R., et al., 2012. Outdoor air pollution and incidence of ischemic and hemorrhagic stroke: a small-area level ecological study. Stroke, 43 (1), 22-27.

Mazaheri Tehrani, A., Karamali, F., and Chimehi, E., 2015. Evaluation of 5 air criteria pollutants Tehran, Iran. International archives of health sciences, 2, 95-100.

Middleton, N., et al., 2008. A 10-year time-series analysis of respiratory and cardiovascular morbidity in Nicosia, Cyprus: the effect of short-term changes in air pollution and dust storms. Environmental health, 7 (1), 39.

Qian, Z., et al., 2007. Association of daily cause-specific mortality with ambient particle air pollution in Wuhan, China. Environmental research, 105 (3), 380-389.

Qorbani, M., and Yunesian, M., 2010. Study designs in air pollution epidemiology. Iranian journal of epidemiology, 5, 44-52.

Rezaei, S., et al., 2016. The effect of air pollution on respiratory disease visits to the emergency department in Kerman, Iran. Journal of health and development, 4, 306-3014.

Roushan, G.R., Negahban, S., and Mirkatouli, J., 2009. Impact of air pollution on climate fluctuations in Tehran city. Journal of Environmental Sciences, 7, 173-192.

Santus, P., et al., 2012. How air pollution influences clinical management of respiratory diseases. A case-crossover study in Milan. Respiratory research, 13 (1), 95.

Shahi, A.M., et al., 2014. The effects of air pollution on cardiovascular and respiratory causes of emergency admission. EMERGENCY-an academic emergency medicine journal, 2, 107-114.

Shamsipour, M., et al., 2014. A framework for exploration and cleaning of environmental data-Tehran air quality data experience. Archives of Iranian medicine, 17 (12), 821-829.

Spix, C., et al., 1993. Air pollution and daily mortality in Erfurt, East Germany, 1980-1989. Environmental health perspectives, 101 (6), 518. 
Stieb, D.M., et al., 2003. Meta-analysis of time-series studies of air pollution and mortality: update in relation to the use of generalized additive models. Journal of the Air \& Waste Management Association, 53 (3), 258-261.

Sun, Q., Hong, X., and Wold, L.E., 2010. Cardiovascular effects of ambient particulate air pollution exposure. Circulation, 121 (25), 2755-2765.

Vahedian, M., et al., 2017. Ambient air pollution and daily hospital admissions for cardiovascular diseases in Arak, Iran. ARYA atherosclerosis, 13, 117-134.

Wang, Y., and Pham, H., 2011. Analyzing the effects of air pollution and mortality by generalized additive models with robust principal components. International journal of system assurance engineering and management, 2 (3), 253-259.

WHO (2018). Ambient (outdoor) air quality and health. 2016. [Updated Februry 2018, Cited 25, Mar 2019]; Available from: https://www.who.int/en/news-room/fact-sheets/ detail/ambient-(outdoor)-air-quality-and-health.
Wong, T.W., et al., 2002. Associations between daily mortalities from respiratory and cardiovascular diseases and air pollution in Hong Kong, China. Occupational and environmental medicine, 59 (1), 30-35.

Yamamoto, S.S., et al., 2014. A systematic review of air pollution as a risk factor for cardiovascular disease in South Asia: Limited evidence from India and Pakistan. International journal of hygiene and environmental health, 217 (2-3), 133-144.

$\mathrm{Yu}$, I.T.S., et al., 2012. Effect of ambient air pollution on daily mortality rates in Guangzhou, China. Atmospheric Environment, 46, 528-535.

Zeng, Q., et al., 2015. Acute effects of $\mathrm{SO}_{2}$ and $\mathrm{NO}_{2}$ on mortality in the six cities of China. Zhonghua yu Fang yi Xue za Zhi [Chinese journal of preventive medicine], 49, 1085-1091.

Zhang, Y., et al., 2006. Ozone and daily mortality in Shanghai, China. Environmental health perspectives, 2, 1227-1232. 